\title{
Modification of Prandtl Wind Tunnel
}

\author{
Bohua Sun†t, \\ (Received 12 December 2018)
}

Wind tunnels are devices that enable researchers to study the flow over objects of interest, the forces acting on them and their interaction with the flow, which is nowadays playing an increasingly important role due to noise pollution. Since the first closed circuit wind tunnel with variable cross-section was built in Göttingen, its Prandtl configuration has little change. The wind tunnel with Prandtl configuration has four corners and vanes, more than $50 \%$ of the total pressure loss are caused by the corners and vanes. How to reduce the total pressure loss is a world class problem in the wind tunnel design. This study attempts to propose a novel configuration of wind tunnel, where the corners have been replaced by semi-circular tunnel. Sun wind tunnel 2 has only two corners and vanes, while Sun wind tunnel 1 has no corners and vanes at all. It is expected the new wind tunnel can reduce the total pressure loss from $50 \%$ to $10 \%$.

Key words: Wind tunnel, Prandtl's configuration, corners, vortex, turbulence, pressure loss

\section{Introduction}

A wind tunnel is a tool used in aerodynamic research to study the effects of air moving past solid objects. Wind tunnel enable researchers to study the flow over objects of interest, the forces acting on them and their interaction with the flow. It has broad applications from aerospace to industrial, from high rise building to automobiles and even from sports and art animations as well. It is easy to be intrigued by application that pervades so many aspects of peoples' daily lives

It was F. H. Wenham (1824-1908) who invented the first enclosed wind tunnel in 1871. K. Tsiolkovsky built an open-section wind tunnel with a centrifugal blower in 1897 . The Wright brothers' use of a simple wind tunnel in 1901 to study the effects of airflow over various shapes while developing their Wright Flyer was in some ways revolutionary. In France, G. Eiffel (1832-1923) built his first open-return wind tunnel in 1909 shown in Fig.1. In 1909 Ludwig Prandtl Prandtl et al. (1969) in G̈̈tingen built a closed circuit wind tunnel with various cross-section shown in Fig.2. Since then the two configurations shown in Fig.3 are coexisting in the wind tunnel design, while the Prandtl configuration dominates the wind design due to its advantages. In this article, we will focus on the design of Prandtl wind tunnel.

This article revisited Prandtl wind tunnel and propose a novel configuration. To make the paper self-contained, the paper is organised as follows. After this introduction, in Section 2 the Prandtl wind tunnel is highlighted with discussion of its both advantages and disadvantages; in Section 3, the main sources of total pressure loss is discussed from perspectives of turbulent flow separation around corners and induced drag of vanes. In

$\dagger$ The Sunshine House, Newlands, Cape Town, South Africa

$\ddagger$ Email address for correspondence: sunbohua@yahoo.com 


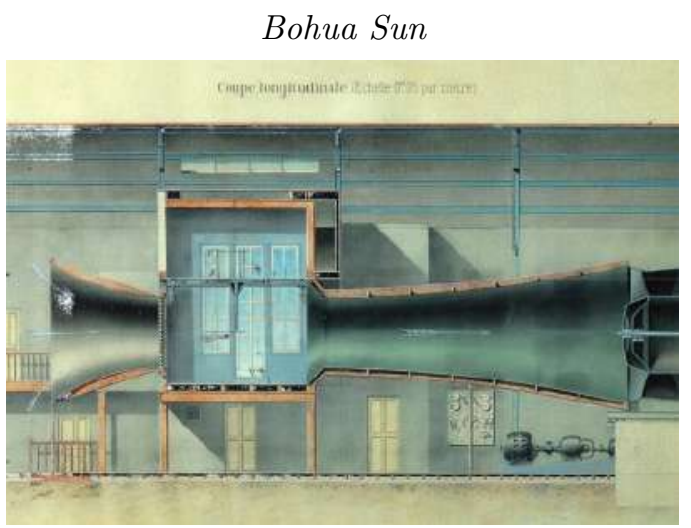

Figure 1: Eiffel wind tunnel (open circuit) in Paris (1909). A cross-section drawing of the wind tunnel with the 2-meter test section in the Eiffel Laboratory at Auteuil Damljanović (2012)

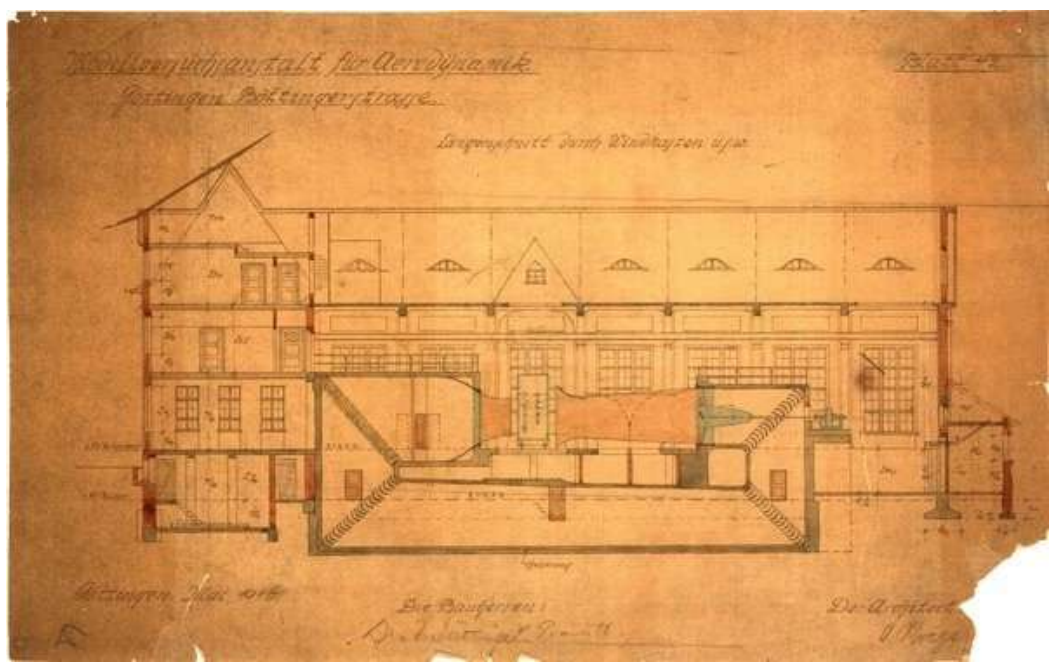

Figure 2: Prandtl wind tunnel (closed circuit) in Göttingen (1917)

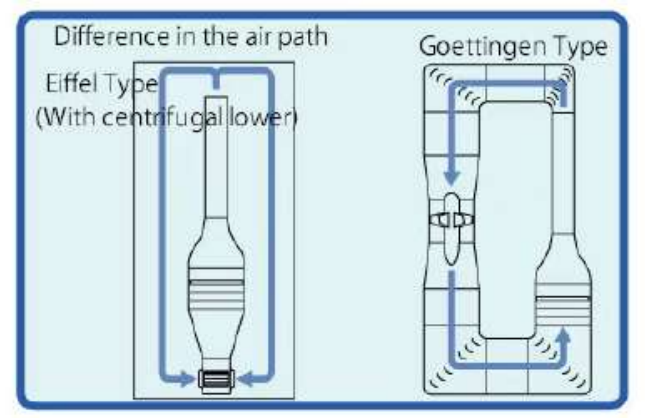

Figure 3: Eifel (open) and Göttingen (closed circuit) wind tunnel configuration 


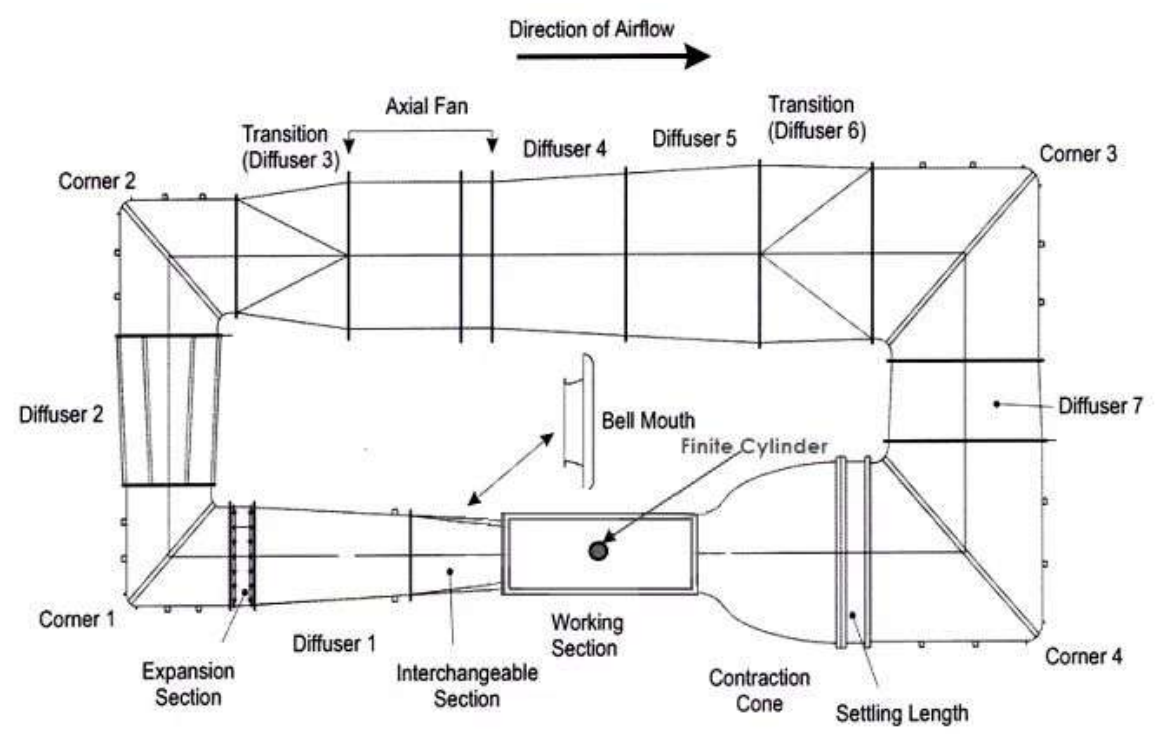

Figure 4: Wind tunnel with Prandtl's configuration

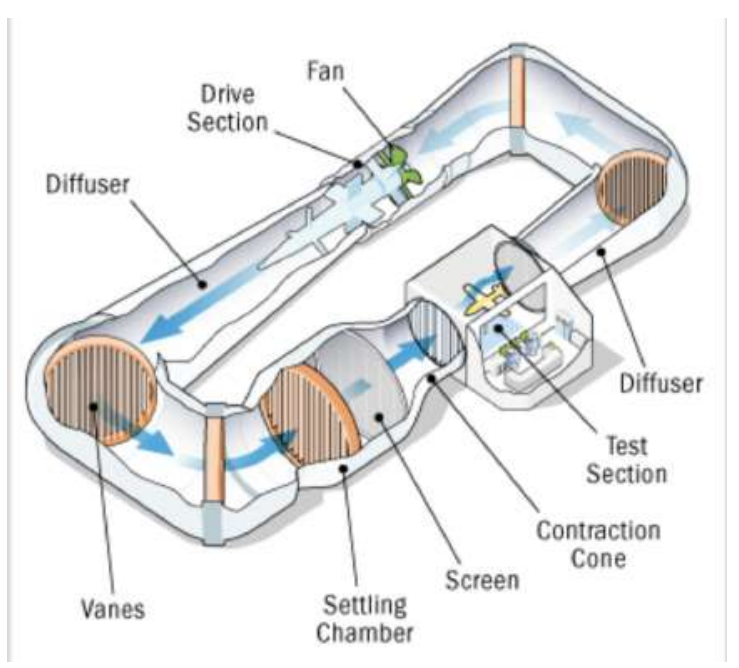

Figure 5: Perspective drawing of a wind tunnel

Section 4 a novel configuration of wind tunnel have been proposed. Finally, section 5 concludes the paper with perspectives.

\section{Prandtl wind tunnel}

The general layout of the Prandtl wind tunnel is shown in Figure 4 below Prandtl et al. (1969). and relevant perspective drawing is shown in 4,

Many of the large research wind tunnels are closed return tunnels. Main components of the wind tunnel are consist of: the contraction zone and the settling chamber. The 


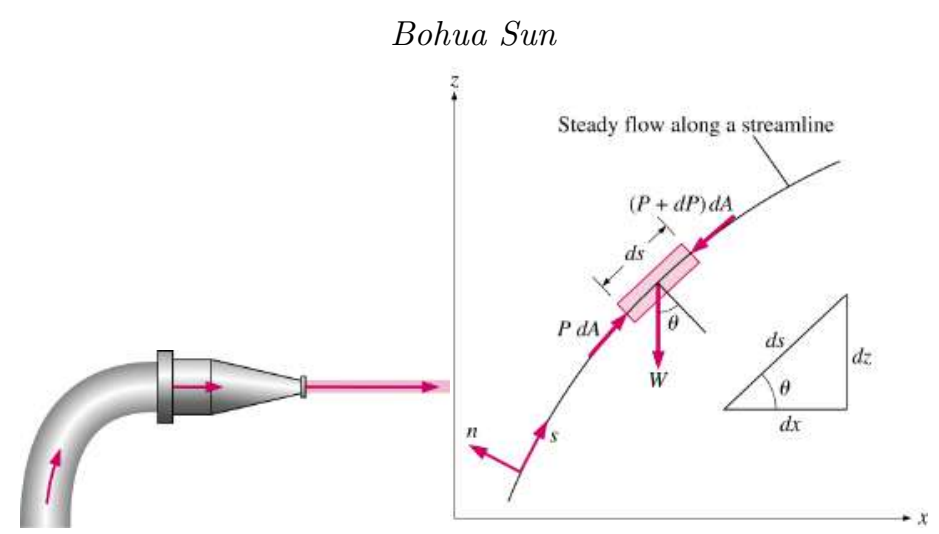

Figure 6: Curve of corner accelerates fluid particle, from Cengel \& Cimbala (2010)

other crucial component is of course the power plant. The remainder of the components just serve the purpose of closing the circuit while minimising the pressure loss (ed).

In the closed return tunnel, air is conducted from the exit of the test section back to the fan by a series of turning vanes. Exiting the fan, the air is returned to the contraction section and back through the test section. Air is continuously circulated through the duct work of the closed return tunnel. The arrows on the figure denote the flow of air through the wind tunnel. In the other major tunnel design, the open return tunnel, air that passes through the test section is gathered from the room in which the tunnel is located.

The closed return tunnel has some advantages and some disadvantages relative to the open return tunnel. Advantages of the closed return tunnel are

(a) Superior flow quality in the test section. Flow turning vanes in the corner and flow straighteners near the test section insure relatively uniform flow in the test section.

(b) Low operating costs. Once the air is circulating in the tunnel, the fan and motor only needs to overcome losses along the wall and through the turning vanes. The fan does not have to constantly accelerate the air.

(c) Quiet operation relative to an open return tunnel. and disadvantages of the closed return tunnel are

(a) Higher construction cost because of the added vanes and ducting.

(b) Inferior design for propulsion and smoke visualization. The tunnel must be designed to purge exhaust products that accumulate in the tunnel.

(c) Hotter running conditions than an open return tunnel. Tunnel may have to employ heat exchangers or active cooling.

\section{Main source of total pressure loss}

Prandtl closed circuit wind tunnels require having four corners, which are responsible for more than $50 \%$ of the total pressure loss. The most critical contribution comes from the corner 1 because it introduces about $34 \%$ of the total pressure loss (ed). Curve of the corners cause the fluid particle acceleration. Assuming the flow inside a wind tunnel is steady flow, ie., $\partial V / \partial t=0$, thus $V=V(s)$, and the acceleration in the s-direction becomes $a_{s}=\frac{d V}{d t}=V \frac{d V}{d s}$, where $V=d s / d t$ if we are following a fluid particle as it moves along a streamline. Therefore, acceleration in steady flow is due to the change of velocity with position. The accelerated fluid will cause velocity gradient that make the formation of circulating fluid structures, called vortices Cengel \& Cimbala (2010).

To reduce the pressure loss and to improve the flow quality at the exit, corner vanes must be added. The vanes and corners will cause the flow severation (shown in Figure 7). 

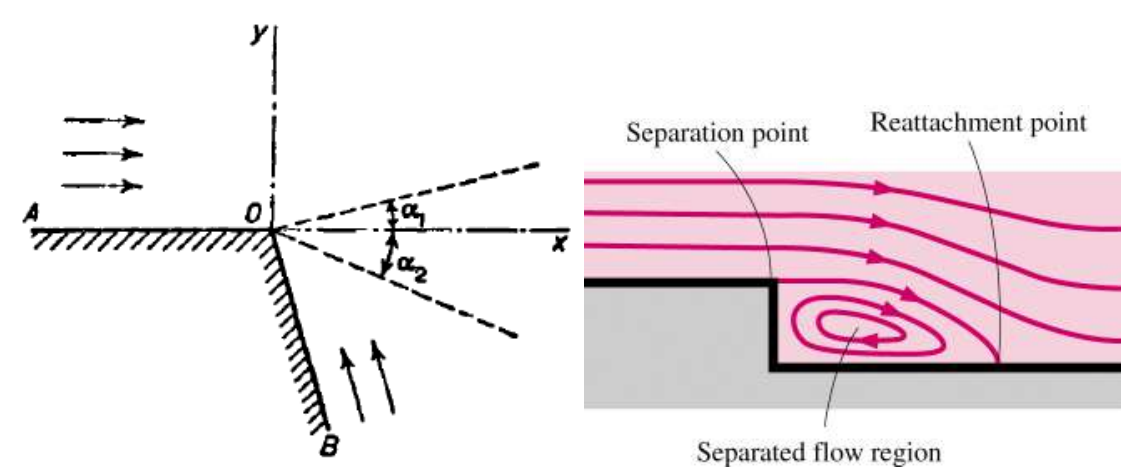

Figure 7: Flow separation around a corner, from Landau \& Lifshitz (2012); Cengel \& Cimbala (2010)

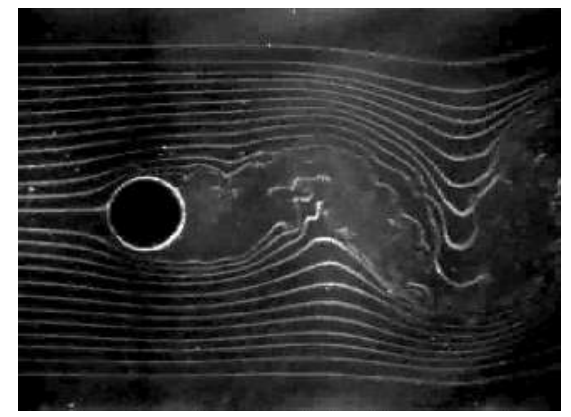

Figure 8: Karmá vortex, from Cengel \& Cimbala (2010)

The important consequence of flow severation is the formation of vortices, in the wake region. Those vortices will eventually transit to turbulence as the increase of flow velocity and acceleration.

The mechanism behind of the total pressure loss is due to the energy dissipation in turbulent flow generated by the corners. The turbulent flow is within the line of $\alpha_{1}$ and $\alpha_{2}$, for flow around a right angle are $\alpha_{1}=5^{0}, \alpha_{2}=10^{0}$. We may menyion that the difference between the fluid pressures on the two sides of the turbulent region is very small. For example, in flow around a right angle it is found that $p_{1}-p_{2}=0.003 \rho U^{2}$, where $U$ is the velocity of mean stream (along AO). $p_{1}$ the pressure in that stream and $p_{2}$ the pressure in the stream along BO (§36, Landau \& Lifshitz (2012)).

Another sources of total pressure loss is the induced drag of vanes. The induced drag is formed by the drag due to the dissipation of energy in the thin turbulent wake. Since the induced drag is proportional to the surface area, therefore more vanes will have more induced drag, which leads more total pressure loss (§47, Landau \& Lifshitz (2012)).

The vortices will forms a Karmán vortex which will make the vanes oscillating and generates noise pollution How to reduce the total pressure loss is a world class problem in the design of wind tunnel.

\section{New configuration of closed circuit wind tunnel}

In order to reduce the total pressure loss and acoustic noise level, it would be a natural attempts to remove corners, namely to replace corners by semi-circular closed. We like 


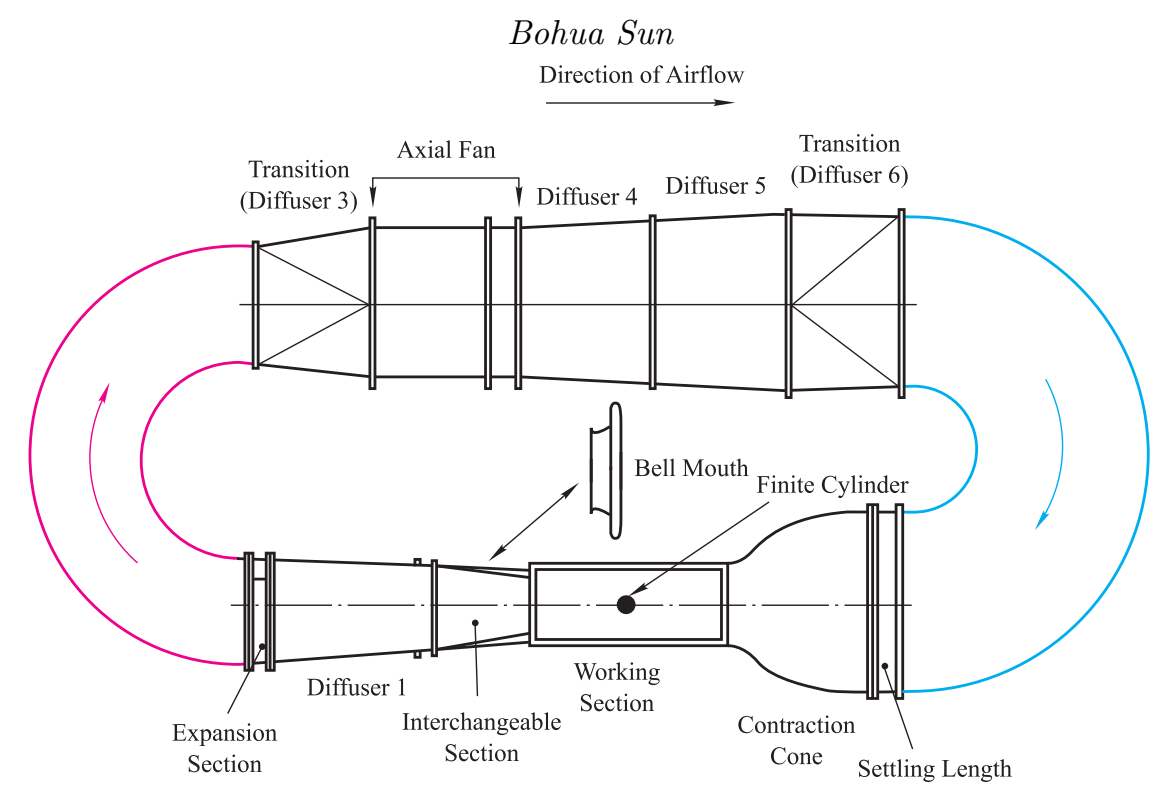

Figure 9: Sun wind tunnel 1

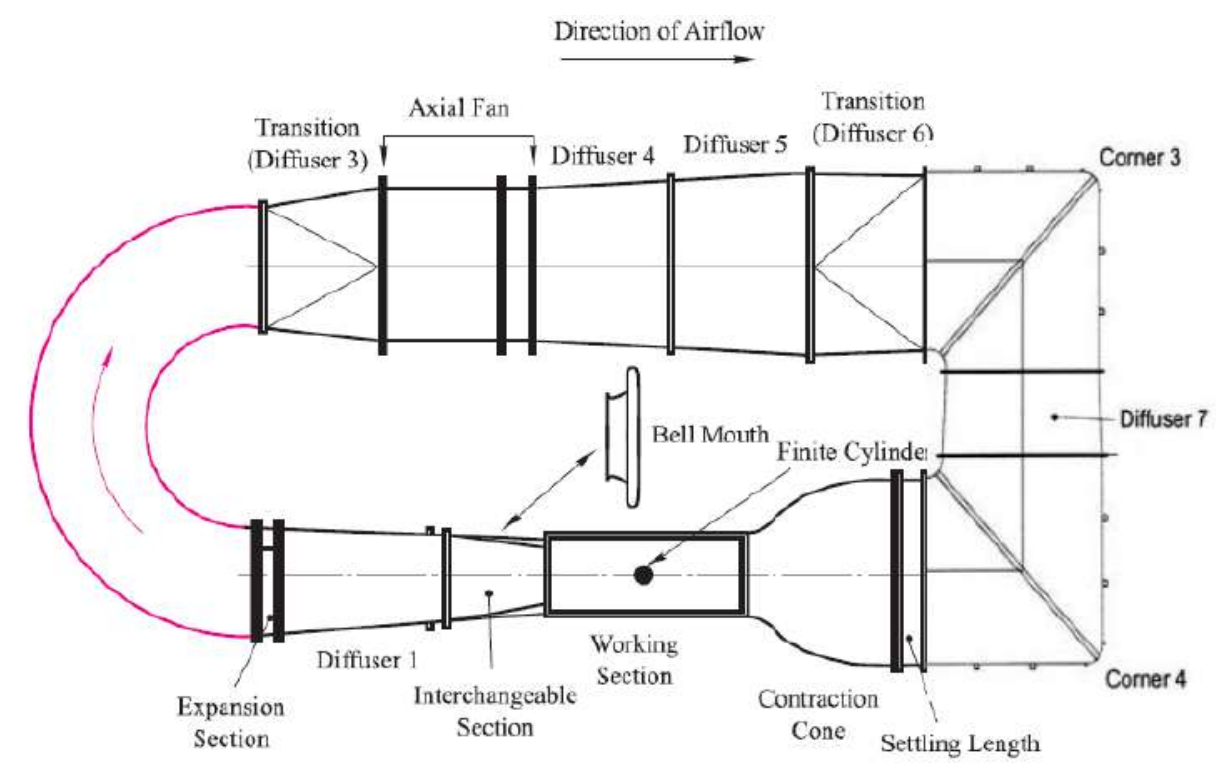

Figure 10: Sun wind tunnel 2

to propose two optional configurations shown in Figure 9 and 10, respectively. The wind tunnel in Figure 9 can be called vanes-free wind tunnel. For convince, the new wind tunnel is called Sun wind tunnel.

It is clear that above two new configurations can definitely reduce the total pressure and energy loss because of their smooth circular curve. It is expected the new configuration will improve the total pressure loss from $50 \%$ to $10 \%$. 


\section{Conclusions}

This study has attempted to propose a novel design of wind tunnel, which can reduce the total pressure and energy loss, and acoustic noise level as well. This is a first part of my research on wind tunnel design. In the future, we will focus on simulation of the proposed wind tunnel Figure 9 and Figure 10, and will build one wind tunnel if financial supports are available and feasible based on Sun Wind Tunnel 1 in Figure 9.

\section{REFERENCES}

Cengel, Y.A. \& Cimbala, J.M. 2010 Fluid Mechanics Fundamentals and Applications. McGraw-Hill.

DamlJanović, D. 2012 Gustave eiffel and the wind: A pioneer in experimental aerodynamics. Scientific Technical Review 62, 3-13.

(ED), N. A. Ahmed 2013 Wind Tunnel Designs and Their Diverse Engineering Applications. InTech.

Landau, L.D. \& Lifshitz, E.M. 2012 Fluid Mechanics. Elsevier.

Prandtl, L., Oswatitsch, K. \& Wieghardt, K. 1969 Führer durch die Strömungslehre. Friedr. Vieweg+Sohn. 